\title{
Influence of the Doppler effect on radiative transfer in a spherical plasma under macroscopic motion of substance
}

\author{
N. I. Kosarev \\ Siberian Federal University, Institute of Non-ferrous Metals and Materials Science, \\ Department of Fundamental Science Education, \\ Krasnoyarsk, 660025, Russia
}

\begin{abstract}
The non-LTE radiative transfer in spherical plasma containing resonantly absorbing of light ions has been studied numerically under conditions of macroscopic motion of substance. Two types of macroscopic motion were simulated: radial expansion and compression (pulsation) of spherical plasma; rotation of plasma relative to an axis of symmetry. The calculations of absorption line profile of transmitted broadband radiation and the emission line profile were performed for the optically dense plasma of calcium ions on the resonance transition with wavelength $397 \mathrm{~nm}$. Numerical results predict frequency shifts in the emission line profile to red wing of the spectrum for radial expansion of the plasma and to blue wing of the spectrum for the plasma compression at an average velocity of ions along the ray of sight equals to zero. The width of the emission line profile of a rotating plasma considerably exceeds the width of the profile of the static plasma, and the shift of the central frequency of resonance transition from the resonance frequency of the static plasma gives a linear velocity of ion motion along a given ray trajectory in units of thermal velocity. Knowledge of the linear radial velocity of ions can be useful for diagnostic purposes in determining the frequency and period of rotation of optically dense plasmas.
\end{abstract}

PACS numbers: 32.70.Jz, 52.25.Os, 92.60.Vb.

\section{INTRODUCTION}

Investigation of expansion of plasma into vacuum is one of the important problems of astrophysics, plasma physics and laser physics, Ref [1]. The temperature of an ultracold plasma (UP) expanding in a magneto-optical trap was investigated in Ref [2] by the resonance absorption method, and in Ref [3] the fluorescence method was used to study the velocity of the ion expansion of UP. Hot pinch plasma produced by pulsed power devices [4] is also of great interest for various applications because it emits thermal X-ray radiation. The properties of such plasma are the high compression and expansion velocities, and the large optical thickness for photons emitted in resonance lines of the highest ionization stages.

Cosmic plasma is also characterized by macroscopic motion. For example the large amount of photometric data indicated the presence of large-scale motion of matter in the stars' shells. Moreover, some variable stars demonstrated pulsating dynamics of their shells, as was reported in Ref [5]. The periodic change in radial velocity of substance, determined from the Doppler shift of the spectral lines relative to their central frequencies, supported this fact. Rotation of matter is one of the types of macroscopic motion, which also characterizes the physical condition of cosmic objects and therefore generates a high interest among astrophysicists in numerous astrophysical applications, as was shown in Ref. [6]. Moreover, rotational dynamics of plasma is an inherent property of experimental installments for controlled thermonuclear fusion problems, Ref. [7].

Attributes of all these types of cosmic and laboratory plasmas are the large optical thickness and motion of substance. Joint influence of these factors on the formation of the emission spectra restricts the possibilities of spectroscopy diagnosing of such plasma. Therefore, the problem of modeling the transport of resonant radiation in plasma with the motion of substance is extremely relevant for various physical applications used spectroscopic diagnostic methods.

In the present article, the transfer of broadband radiation in spherical plasma containing resonantly absorbing of light ions is studied using the method of numerical simulation. Two types of macroscopic motion are modeled. These are the radial expansion of spherical plasma changing to compression (pulsation) and rotation of spherical plasma relative to an axis perpendicular to the 
direction of propagation of an external radiation. This nonstationary problem is described by the rate balance equations for two-level ion populations and by the radiative transfer equation for threedimensional geometry of medium. The numerical solution of the obtained system of integrodifferential equations allowed us to study the influence of the Doppler shift on intensity of absorbed sunlight and radiation emitted by calcium ions at the frequency of the resonance transition ${ }^{2} S_{1 / 2} \leftrightarrow{ }^{2} P_{1 / 2}$ with the wavelength of $\lambda_{0}=397 \mathrm{~nm}$. along different optical paths.

\section{MATHEMATICAL FORMULATION OF THE PROBLEM}

\subsection{Rate balance and radiative transfer equations at macroscopic motion of substance}

Let us describe the mathematical formulation of the problem for non-LTE and non steady-state plasma which contains ions that absorb external electromagnetic radiation and re-emit it in a single optical depth resonance line. Therefore, we can use the standard two-level model of the ion, including the ground level 1 and the excited one 2. The rate balance equations for the ion populations $N_{1}$ and $N_{2}$ on the corresponding levels 1 and 2 have the form

$$
\begin{aligned}
& \frac{d N_{1}(\mathbf{r}, t)}{d t}=-B_{12} J(\mathbf{r}, t) \cdot N_{1}(\mathbf{r}, t)+\left(B_{21} J(\mathbf{r}, t)+A_{21}\right) \cdot N_{2}(\mathbf{r}, t), \\
& \frac{d N_{2}(\mathbf{r}, t)}{d t}=-\left(B_{21} J(\mathbf{r}, \mathrm{t})+A_{21}\right) \cdot N_{2}(\mathbf{r}, \mathrm{t})+B_{12} J(\mathbf{r}, \mathrm{t}) \cdot N_{1}(\mathbf{r}, \mathrm{t}),
\end{aligned}
$$

were $B_{12}, B_{21}$ and $A_{21}$ are Einstein coefficients for the probabilities of stimulated radiative excitation of normal ions, de-excitation and spontaneous decay of excited levels, consequently. In equations (1) and (2), the coefficient $J(\mathrm{r}, t)$ is the averaged by angle- and frequency-integrated radiation intensity at the point $r$, at the time $t$, which is formed by external and own (re-emitted) radiation

$$
J(\mathbf{r}, \mathrm{t})=\frac{1}{4 \pi} \int_{0}^{2 \pi} d \varphi \int_{0}^{\pi} \sin (\theta) d \theta \int_{-\infty}^{\infty} \Phi(x, \mu ; u) I_{x}(\mathbf{r}, \theta, \varphi, \mathrm{t}) d x
$$

were $x=\left(v-v_{0}\right) / \Delta v_{\mathrm{D}}$ denotes the shift of the frequency $v$ from the central frequency $v_{0}$ in the rest frame, expressed in units of the Doppler width $\Delta v_{\mathrm{D}}=v_{0} v_{\mathrm{th}} / c ; \Phi(x, \mu ; u)$ is the line profile which for the case of macroscopic motion of substance is broadened by the Doppler effect and the macroscopic velocity of the ions. In this case the normalized line profile in the observer's frame is [5]

$$
\Phi(x, \mu ; u)=\pi^{-1 / 2} \delta^{-1}(\mathbf{r}, \mathrm{t}) \cdot \exp \left\{-[x-\mu u]^{2} / \delta^{2}(\mathbf{r}, t)\right\}
$$

were $\mu=\cos \theta, \theta$ is the angle between the direction of propagation of the photon and the normal directed to the observe; coefficient $u(\mathbf{r}, t)=v(\mathbf{r}, t) / v_{\text {th }}$, were $v(\mathbf{r}, t)$ is the macroscopic ion velocity with respect an observe positioned outside the plasma sphere, $v_{\text {th }}$ is the mean thermal ion velocity corresponding to the standard Doppler width $\Delta v_{\mathrm{D}}$; the coefficient $\delta(\mathbf{r}, \mathrm{t})=\left(\Delta v_{\mathrm{D}}+\left|v(\mathbf{r}, t) / \lambda_{0}\right|\right) / \Delta v_{\mathrm{D}}$ depends on the temperature and the ion velocity at a given point $r, \lambda_{0}=c / v_{0}$ is the wavelength, $\mathrm{c}$ is the speed of light. The line profile $\Phi(x, \mu ; u)$ depends on the time through the coefficients $\delta(\mathbf{r}, t)$ and $u(\mathbf{r}, t)$ if we assume that the velocity $v(\mathbf{r}, t)$ at any point in the medium can change with time. 
To calculate the triple integral in Eq. (3) for each spatial point of the three-dimensional volume, one needs to have the spectral radiation intensity $I_{x}(\mathbf{r}, \theta, \phi, t)$ formed by external radiation and internal sources of photons. This spectral intensity $I_{x}(\mathbf{r}, \theta, \phi, t)$ at point $\mathbf{r}$ in the direction determined by angles $\theta$ and $\phi$, in quasi-stationary approximation and for complete frequency redistribution case is determined by solving the transfer equation

$$
\frac{d I_{x}}{d \tau}=\Phi(x, \mu, u) \cdot\left[S-I_{x}\right]
$$

Here the source function $S=\eta(\mathbf{r}, t) / \chi(\mathbf{r}, t)$ determined by the emission $\eta$ and absorption $\chi$ coefficients on the frequency $v_{0}$. The dimensionless quantity $d \tau=\chi d r$ is an infinitesimal increment of the optical thickness of an emitting and absorbing layer of a plasma having thickness $d r$. The coefficients $\chi$ and $\eta$ implicitly depend on the time $t$ through the populations of the ground $N_{1}$ and excited $N_{2}$ levels of the resonance transition. They are determined in this way

$$
\chi(\mathbf{r}, t)=\alpha_{12} \cdot\left[N_{1}(\mathbf{r}, t)-\frac{g_{1}}{g_{2}} N_{2}(\mathbf{r}, t)\right], \quad \eta(\mathbf{r}, t)=\frac{2 h v_{0}^{3}}{c^{2}} \cdot \alpha_{12} \frac{g_{1}}{g_{2}} \cdot N_{2}(\mathbf{r}, t) .
$$

Here $\alpha_{12}=B_{12} h v_{0} /\left(4 \pi \cdot \Delta v_{\mathrm{D}}\right)$, where the Doppler width $\Delta v_{\mathrm{D}}$ appeared in the denominator in view of the normalization of the absorption line profile, Eq.4; $g_{1}$ and $g_{2}$ are the statistical weights of levels, $\mathrm{h}-$ is the Plank constant.

The equations (1)-(6) must be supplemented by initial and boundary conditions. The first, assuming the concentration of ions at the initial instant of time $t_{0}=0$ to be equal $N_{0}$ and uniform ion distribution in a plasma, have the form

$$
N_{2}(\mathbf{r}, 0)=0, \quad N_{1}(\mathbf{r}, 0)=N_{0} .
$$

The boundary conditions will correspond to the case when the incident (from outside) radiation irradiates the one boundary of the spherical plasma, the left boundary on Fig. 1, and there is no incident radiation at the right boundary of the sphere. We also believe that external radiation of intensity $I_{S}^{0}$ is broadband. It has axial symmetry and over the spectral line is independent of frequency. Then the boundary condition for Eq. (5) on the irradiated (left) boundary of the sphere can be written in the form

$$
I_{x}(\mathbf{R}, \theta, \varphi, t)=\left\{\begin{array}{ll}
I_{S}^{0}, & \text { if } \theta=0, \\
0, & \text { if } \theta \neq 0
\end{array},\right.
$$

where $\mathbf{R}$ denotes the radius vector of the irradiated surface of the sphere, Fig. 1(a).

We also introduce the total optical thickness $\tau_{0}=2 R_{0} \chi\left(R_{0}, t_{0}\right)$ of the medium at the central frequency $v_{0}$ along the diameter of the sphere for a static substance, where $R_{0}$ is the radius of the static sphere at the initial instant of time.

\subsection{Rotating of the sphere around an axis of symmetry}

Figure 1 shows the geometry of the problem for the case of rotation of the sphere around the axis of symmetry perpendicular to the direction of incident radiation. The plasma sphere of radius $R_{0}$ 
rotates with angular velocity $\Omega_{0}=V_{0} / R_{0}$ relative to the $Y$ axis, Fig. 1, counterclockwise, where $V_{0}$ is the linear velocity of the points of the medium lying on the circle of radius $R_{0}$, Fig. 1(b). Then, at each point of the spherical volume, linear velocity of ions, located at the distance $r$ from the axis of rotation $Y$ and moving on the circle of the radius $|r|$, Fig. 1 (a), can be given by the following expression

$$
v(\mathbf{r}, t)=\frac{V_{0}}{R_{0}} \cdot|r|
$$

Here the dependence of the velocity on time indicates that the angular velocity $\Omega_{0}$ can also change with time.

\subsection{Radial pulsation of a sphere}

The radial expansion and contraction of the sphere are described by the equation (8), but now the radial ion velocity at a point with radius vector $\mathbf{r}$ is determined by the expression $v(\mathbf{r}, t)=V(t) / R \cdot \mathbf{r}$, where $V(t)$ is velocity of ions on the boundary of the sphere of radius $R$. Then velocity $v(\mathbf{r}, t)$ is a vector indicating that ions move alone the normal from the center of the sphere to the outside or to its center. In the case of pulsating plasma, the ion velocity was modeled by the following harmonic dependence

$$
V(t)=V_{0} \cdot \sin \left(\frac{t}{T_{0}} \cdot \frac{\pi}{2}\right)
$$

Here $T_{0}$ is the time corresponding to a quarter of the period of the function $\sin (\xi)$, and $V_{0}$ is the peak velocity of the sphere's boundary at the time $T_{0}$, for which the argument of the sinus function is equal to unit. The time range between 0 and $4 \times T_{0}$ fully covers the first phase of expansion and compression. The range $0 \leq t \leq 2 T_{0}$ corresponds to the phase of the radial expansion of the sphere, for which the function $\sin (\xi)$ and, consequently, the velocity $V(t)$ take positive values. Accordingly, the sphere's radius increases during this period of time. At times between $2 T_{0}<t \leq 4 T_{0}$, the velocity $V(t)$ becomes negative and, consequently, the sphere is radially compressing. Taking into account Eq. (9) the radial velocity of ions at the point $\mathbf{r}$ will have the form

$$
v(\mathbf{r}, t)=\frac{\left(V_{0} \cdot \sin \left(\frac{t}{T_{0}} \cdot \frac{\pi}{2}\right)\right)}{R} \cdot \mathbf{r} .
$$

When ions move in the radial direction, the radius of the sphere and, consequently, its volume change. Therefore, the total ion concentration $N(r, t)=N_{1}(r, t)+N_{2}(r, t)$ at any point of the medium $\mathbf{r}$ will depend on time. To take this fact into account we use the dependence between the sum $N_{1}(\mathbf{r}, t)+N_{2}(\mathbf{r}, t)$ and the ion concentration at the initial instant of time $N_{0}$

$$
N_{1}(\mathbf{r}, t)+N_{2}(\mathbf{r}, t)=N_{0} \cdot \frac{R_{0}^{3}}{\left(R_{0}+v(\mathbf{r}, t) \cdot t\right)^{3}} .
$$


Equation (11) complements the system of Eqs. (1)-(7), (9), (10) and shows that if the sphere expands, then the velocity $v(\mathbf{r}, t)$ is positive and at each point of plasma with the coordinate $r$ has the radial direction from the center of the sphere to the outside. Therefore, the ion concentration per unit volume decreases with time. At compression, on the contrary, the ion velocity $v(\mathbf{r}, t)$ is negative and at any point of a plasma has the direction radially to the center of the sphere. The ion concentration now increases, returning to its original value $N_{0}$.

\subsection{Algorithms for the numerical solving of a system of integrodifferential equations}

The system of Eqs. (1)-(7) has integrodifferential nature, because there is the coefficient $J(\mathbf{r}, t)$, what enters into the rate balance equations (1) and (2). This coefficient represents a triple integral of the spectral intensity of the radiation $I_{x}(\mathbf{r}, \theta, \phi, t)$. The latter itself depends on the level populations through the absorption coefficient $\chi(\mathbf{r}, t)$ and the source function $S(\mathbf{r}, t)$. To calculate the spectral intensity numerically in a set of angles, frequencies, and spatial points, we used a formal solution of the transfer equation in integral form [5]

$$
I_{0}=I_{\mathrm{M}} \cdot \exp \left(-\Phi \Delta \tau_{\mathrm{M}}\right)+\int_{0}^{\Delta \tau_{M}} S(\tau) \cdot \exp \left[-\Phi\left(\Delta \tau_{\mathrm{M}}-\tau\right)\right] d \tau
$$

for any segment of integration $M O$, along the ray path from point $M$ to point $O$. In the expression (12), the optical thickness $\Delta \tau_{\mathrm{M}}$ is calculated from expression $\Delta \tau_{\mathrm{M}}=\int_{O}^{M} \chi(\mathbf{r}, t) d s$, and the value of the spectral intensity $I_{0}$ at some nodal point $\mathrm{O}$, is calculated with using a known value $I_{M}$ at the point $M$. When all nodal values of the spectral intensity were obtained at a fixed time, integration in expression (3) over the frequency, angular and spatial variables was carried out numerically, using specially selected quadrature formulas [8]. Now, if the right-hand side of equations (1) and (2) is calculated at each time, they represent a system of ordinary differential equations (ODE) of the form

$$
\frac{d N_{i}(t)}{d t}=F_{\mathrm{i}}\left[N_{\mathrm{i}}(t), t\right], t \in[0, T], i=1,2,3, \ldots N_{\mathrm{D}}
$$

The dimension of this system of equations $N_{\mathrm{D}}$ is equal to the product of the number of ionic levels and the number of spatial nodes of the three-dimensional sphere. In paper [8] numerical methods for solving the ODE system are presented for three-dimensional steady-state media. Macroscopic motion of substance was taken into account in the absorption line profile, Eq. (4), introducing in the numerical algorithm arrays of coefficients $\delta(\mathbf{r}, t)$ and $u(\mathbf{r}, t)$, in which the directions and the values of velocities in the spatial nodes of the three-dimensional grid were determined according to Eqs. (8), (10). The constructed numerical model of radiative transfer in the condition of macroscopic motion of substance make it possible to calculate the populations of two-level ions and the spectral radiation intensity $I_{x}(\mathbf{r}, \theta, \phi, t)$ at times including a sufficiently large number of pulsation phases of the spherical plasma, or at linear ion velocities $V_{0}$ reaching ten of thermal velocities. The results of modeling of absorption of broadband and laser radiation at expansion of gas and plasma are presented in articles [9-14]. Next, we present results that take into account both the radial pulsation and the rotation of the spherical plasma. 


\subsection{Parameters of model}

Using the developed numerical algorithms the problem of absorption of sunlight and emissing of radiation by the sphere plasma of calcium ions on the resonance transition with $\lambda_{0}=397 \mathrm{~nm}$ is solved. This formulation of the problem relates to research of the properties of the upper atmosphere and magnetosphere of the Earth by injecting into it artificial luminous clouds, Ref. [15]. Such a model object of research, perhaps, does not describe a real astrophysical object, but from the theoretical point of view demonstrates the influence of the Doppler effect on the formation of absorption and emission spectra by optically dense media, in the presence into them of the certain type of macroscopic motion of substance.

For the mentioned above resonance transition in the calcium ion, the value $I_{\mathrm{S}}^{\mathrm{O}}$ is $1.23 \times 10^{10} \mathrm{erg} \cdot \mathrm{cm}^{-2} \cdot \mathrm{s}^{-1} \cdot \mathrm{cm}^{-1}$, Ref. [16]. According to the experimental data of Ref. [15], we can assume: the kinetic temperature of the injected vapor is equal to $T_{0}=1160^{\circ} \mathrm{K}$ that corresponds to the thermal ion velocity $v_{\text {th }}=6.92 \times 10^{4} \mathrm{~cm} / \mathrm{s}$; the geometry of artificial clouds can be considered spherical, and the characteristic diameter of the cloud is $D_{0} \approx 10^{6} \mathrm{~cm}$. In this case, the initial atomic concentrations of the injected chemical element reached values $N_{0} \approx 10^{7}-10^{8} \mathrm{~cm}^{-3}$. For the ion concentration equals $N_{0}=4.85 \cdot 10^{6} \mathrm{~cm}^{-3}$ and at the initial diameter of the static cloud equals $D_{0}=2 R_{0}=10^{6} \mathrm{~cm}$, we obtain for the static optical thickness at the central frequency of the ion transition the value equal to $\tau_{0}=25$. The peak velocity of expansion (compression) at the time when the argument of the sine function in Eq. 1 is equal to +1 (respectively, for compression -1), equal to $V_{0}=5 \times v_{\text {th }}=3.46 \times 10^{5} \mathrm{~cm} / \mathrm{s}$. For the case of rotation of the plasma sphere, the velocity $V_{0}$ also did not exceed the values $5 \times v_{\text {th }}$ and was assumed to be constant. Then the velocity $V_{0}$ in Eq. (8) also did not depend on time.

\subsection{Rotation of the spherical plasma}

The data shown correspond to the absorbed and emitted radiation propagating in the central impact plane of the sphere, Fig. 1 (b). The central impact plane represents the cross-section of the sphere by the plane crossing the $X$ and $Z$ axes [7]. In figure 1 (b), the numbers $1-7$ in the upper semicircle indicate the exit points for radiation propagating along optical paths parallel to the $\mathrm{Z}$ axis and these optical paths are at different distances from the center of the sphere. Each of those optical paths corresponds to different optical thickness for radiation and to different projections of ion linear velocity to the given ray direction. The numbers 1-7 of the lower semicircle indicate the exit points of emission from the medium perpendicular to the direction of sunlight from the irradiated side of the sphere.

Figure 2 shows the frequency form of transmitted solar intensity passing through the plasma on the different optical tracks of the upper semicircle of the central impact plane. For any optical path, except the one presented by line 4, the cosine of the angle $\theta$ between the projections of ion velocities and the direction of light propagation in Eq. (4) is less than zero. Therefore, the ion absorption line profiles are shifted to the red wing of the spectrum. Consequently, the maxima of the absorption profiles for transmitted sunlight will also be shifted in the same wing of the spectrum. The largest shift of the central frequency in transmitted radiation corresponds to the track, which is farthest from the diameter (see line 1), because for it projections of ion velocities on the direction of light propagation take the greatest value with a minus sign. As the geometric path approaches the diameter of the sphere, the projections of ion velocities to the direction of sunlight 
decrease (line 2 and 3 of Fig. 2). Therefore, the central frequency in transmitted approaches the frequency corresponding to the central frequency $v_{0}$ for static medium.

For the ray track passing through the diameter of the sphere, solid line 4 in Fig. 2, the velocity direction of ions located at the diameter is perpendicular to that of sunlight propagation. Therefore, the centers of the absorption line profiles for these ions coincide with the central frequency of static medium, but the width of the absorbed profile at the half-depth greatly exceeds the respective width of the static profile. This can be seen by comparing solid curve 4 and dashed one 4'. The latter is obtained for the same path, but in the case of static medium. This difference can be explained by Eq. (4) describing the Doppler profile of the ion absorption line under the condition of macroscopic movement of substance. When the linear velocity $v(\mathbf{r})$ increases the coefficient $\delta(\mathbf{r})$ increases too. This coefficient in Eq. 4 is simultaneously located in the denominator of the constant in front of exponent, and in the denominator of the index of function exp, squared. The coefficient $\delta(\mathbf{r})$ increases with increasing a velocity $v(\mathbf{r})$. Therefore, at any fixed frequency $x$, the coefficient $1 / \delta(\mathbf{r})$ decreases the peak value of the function $\exp$ with increasing velocity. On the other hand, with frequency detuning from the line center, the more a velocity $v(\mathbf{r})$, the more slower the decrease of function exp in the spectrum wings. This is determined by the presence of the coefficient $(1 / \delta(\mathbf{r}))^{2}$ in the index of exponent. Hence, for moving medium, the absorption coefficient increases at all frequencies of the line profile relative to the static medium. And, in this respect, the width of the absorbed line profile at the half-depth for rotating plasma is much larger than the one for static plasma, even for the case when along the optical path the direction of ion velocity is perpendicular to the sunlight direction.

The frequency dependences for radiation emitted by the plasma and passing through different optical paths are shown in Fig. 3 for directions perpendicular to the direction of sunlight [irradiated boundary in Fig. 1(b)]. For lines 1 and 2, the direction of photons' emitting coincides with the projection of ion velocities on these optical paths. Consequently, the centers of the absorption line profiles, as well as the centers of the emission line profiles of ions, are shifted to the blue wing of the spectrum (the cosine of the angle in Eq. (4) is positive). The maxima of the emitted radiation intensity are also shifted to the blue wing of the spectrum. The farther the path from the diameter of the sphere, the larger the shift of intensity maxima from the line center to the blue frequency wing (see lines 1 and 2 in Fig. 3).

Line 3 in Fig. 3 corresponds to the track passing through the sphere's diameter. The projections of ion velocities to the emitting direction are zero for all points along the diameter. This is why the center of the emission line coincides with the central frequency of the ion line $v_{0}$ for static medium along this optical path. However, the full width at half maximum (FWHM) of the emission line is much larger than the value of $F W H M$ for static medium (see solid 3 and dashed 3'). The explanation of this emission line broadening is the same as for increase in the width of the absorption line in the transmitted solar spectrum. As was already noted, the ion absorption coefficient of moving medium is greater than the one of static medium at all frequencies, for all optical tracks including those for which the cosine of the angle $\theta$ between the direction of ion's velocity and the direction of the photons' emission is zero. In turn, the emission spectrum is formed by the source function $S=\eta / \chi$ from the transfer equation (5) where the profiles of the absorption and emission lines coincide.

Dashed lines 1' and 2' in Fig. 3 show the emission intensity for ray directions symmetric to the directions presented by solid lines 1 and 2, respectively, relative to the sphere's diameter, but from its shadow side. They have the symmetric shifts of intensity maxima, but to the opposite (red) frequency wing. In addition to this difference, the amplitudes of their intensities are much smaller than the amplitudes of intensities represented by lines 1 and 2, because of strong absorption of radiation by dense plasma. 
Figure 4 shows the frequency profile for radiation emitted in the sunlight direction from the shadow side of the sphere for different ray tracks. Solid lines 1 and 2 correspond to the upper hemisphere, whereas dashed ones 1' and 2' correspond to the lower hemisphere. The lines of the same name correspond to the tracks that are distant from the sphere's diameter at an equal distance, but in opposite directions. Solid line 3 here corresponds to the optical track passing along the sphere's diameter. For lines 1 and 2 (upper semicircle) the projections of ion velocities to their optical ray are opposite to the direction of photons' emission. Hence, the emission profile is shifted into the red spectral wing. For the optical paths corresponding to the dashed lines 1' and 2' (lower semicircle), the projections of ion velocities coincide with the direction of photons' emission. Therefore, the emission line profiles are shifted to the blue frequency wing. At the same time, the blue frequency shift is completely symmetric to the red shift for the optical tracks having the same numbers and located symmetric relative to sphere's diameter.

We should pay attention to the self-reversal (re-absorption) of the emission line for the optical path represented by line 3 (the diameter of the sphere). Re-absorption is caused by a large optical thickness of the medium at the resonance transition. The phenomenon of re-absorption of the emission spectrum has been studied in detail for static, optically dense media in papers [17-19]. It was shown in these papers that in optically dense media the emission directions opposite to the external radiation are accompanied by the decrease in absorption coefficient. Therefore the escape of photons to these directions is easier. In this case the frequency line profile for such ray paths is not re-absorbed. The propagation of photons along the direction of external radiation, from the shadow boundary of medium, is accompanied by increasing in the absorption coefficient. Hence, for these ray tracks the escape of photons is more difficult in comparison with the opposite emission directions. For this reason, the re-absorption of the spectral profile appears for the radiation emitted from the shadow side of medium.

The behavior of solid line 3 in Fig. 4 qualitatively agrees with the conclusions from papers [1720], but for lines 1, 2, 1', and 2' there is no re-absorption because of an insufficiently large optical thickness of these ray tracks. In addition to the phenomenon of re-absorption, for rotating plasma the frequency shift of the center of emission line profile in the red or blue regions of the spectrum appears.

If we will compare lines 3' (static plasma) and 3 in Fig. 4, which both correspond to the ray track passing along the sphere's diameter, we can note again that the value of $F W H M$ for rotating plasma is significantly larger than the respective $F W H M$ for stationary plasma. This fact can be explained the same way as the increase in $F W H M$ for the emission spectrum in Fig. 3 (line 3).

Having the shift of the emission spectra from the central frequency of the static plasma, Fig. 3, one can determine the direction of the plasma rotation and the linear velocity of the ions, registering radiation along different ray directions. Knowledge of the linear radial velocity of ion motion makes it possible to calculate the frequency and period of rotation of the plasma. The solution of such problems is especially important for astrophysical research [21]. It is not difficult to understand that each ion on the chosen ray direction has the same projection of the linear velocity on this direction, Fig. 1b. Therefore, the value of the frequency shift from the line center, determined by the addition $\mu u$ in the profile of the absorption line (4), is the same along the given ray direction. If the profiles of the ion absorption lines along the ray direction are shifted from the central frequency by the same value, then the emission line profiles of ions will be shifted by the same value. Consequently, the value of detuning the frequency from the line center at the given optical path gives the value of the linear ion velocity in units of thermal velocity $v_{\text {th }}$. If the line profile is strongly reabsorbed, as in the case of the ray directions represented by the curves 3, 3' in Fig.4, the frequency shift of such a profile can be determined using the method of the bisector of absorption [21]. A bisector is a line dividing the reabsorbed profile into two halves with equal equivalent widths.

It should be noted that the thermal velocity of the ions is calculated from the plasma temperature. The latter is fairly accurately determined from the width of the emission line profile for an optically thin medium. However, for an optically dense, rotating plasma, the width of the emission line profile does not reflect its temperature. Consequently, in this case, to determine the kinetic 
temperature of the plasma, the spectroscopic method based on measuring the Doppler profile of the spectral line is unacceptable.

\subsection{Pulsating dynamics of the spherical plasma}

Investigating of the radiative transfer in plasma for the case of pulsating dynamics of sphere, for convenience of numerical calculation, characteristic times of the considered pulsation process were measured in microseconds. Such times correspond to the characteristic retention times of ultracold plasma in MOT, Ref. [2,3]. The optical tracks passed through the center of the sphere.

Figure 5 shows the absorption spectrum of solar radiation at various points of sphere's diameter, starting from the irradiated boundary of plasma to the shadow one. The time moment $t$ equals $T_{0}=1 \mu \mathrm{s}$, for which velocity of the sphere boundary expansion is maximal and equals $V_{0}$. The red spectral wing is more strongly absorbed from the irradiated boundary of the sphere and to its center. It is caused by the shift to the red wing of the absorption line profiles of ions due to the Doppler effect (lines 1-3). Indeed, the direction of solar radiation before the sphere's center is opposite to that of ion velocities and the parameter $\mu$ in Eq. (4) equals -1 , whereas between the center of the sphere and its shadow boundary, the blue wing of the spectrum is more strongly absorbed (lines 46), because now the absorption line profiles of ions shifts to the blue spectral region. Absorption of the blue spectral wing compensates absorption of the red spectral region. Therefore, at the exit from plasma, the absorption spectrum of solar radiation becomes symmetrical in frequency relative to the central frequency of resonance line (line 6).

For the moments of time when expansion of the sphere changes by compression, the view of absorbed spectrum of transmitted solar intensity at different points of the diameter is completely opposite to the lines in Fig. 5. Now the blue spectral region is more strongly absorbed before the sphere's center, while the red one does after the center of the sphere. At the exit from the medium the absorption spectrum of solar radiation again becomes symmetrical in frequency.

For times at which there is no plasma motion, the solar radiation is absorbed symmetrically in frequency relative to the central frequency of the line $v_{0}$ at all spatial points. The contour of the absorption line at the exit from medium has a Doppler frequency form in accordance with Eq. (4) at $u=0$, and its width at half-depth is much less than the width for moving medium.

Figure 6 shows the frequency distribution of radiation emitted by the spherical plasma in the resonance line of the calcium ion $\left(\lambda_{0}=397 \mathrm{~nm}\right)$ to the direction opposite to the sunlight (backward direction; curve 1), to the direction perpendicular to the sunlight (lateral direction; curve 2), and to the direction of the sunlight (forward direction; curve 3). Solid lines 1-3 were obtained at the time of the fastest expansion velocity for the eighth pulsation phase.

When the sphere is expanding, the backward emitted radiation is shifted in frequency to the blue wing of the spectrum, solid line 1 in Fig. 6 . This radiation is formed by excited ions located near the irradiated boundary of the sphere. For these ions the emission line profiles are shifted to the blue spectral wing, and the backward photon escape from the medium is easier relative to the other emission directions [17-20]. When photons are emitting in the lateral direction, the red wing of the spectrum increases, while the blue one attenuates. Nevertheless, intensity of blue photons is higher than that of red photons. When photons are forward emitted (solid line 3 in Fig. 6), expansion of plasma leads to the shift of the spectral profile to the red wing. This occurs because of the following reasons. First, in the region between the entry boundary and the center of medium the forward emitted radiation undergoes the Doppler shift to the red wing of the spectrum. Second, in the region between from the center of the sphere and its shadow boundary the forward emitted radiation is already more strongly absorbed in the blue spectral wing, while the red wing of the spectrum already passes through plasma without significant absorption.

The shape of solid line 3 indicates that when the sphere is expanding, the right wing of the static re-absorbed profile (dashed line 3' in Fig. 4) is weakened and, broadening, shifts to the blue wing of the spectrum. The left wing of the static profile is amplified and, broadening, shifts to the red wing 
of the spectrum. The spectral line generally looks shifted into the red spectral wing. Thus, we have the shift in the maximum of the spectral line profile at an average expansion velocity along the line of sight equals to zero.

The dashed lines 1'-3' in Fig. 6 are obtained for the eighth phase of pulsation of the substance at the moment of time corresponding to the highest compression velocity of the sphere's boundary. As is seen, all lines excluding line 1' demonstrate an almost symmetrical shift of the intensity maximum to the red or blue wing of the spectrum relative to the central frequency. The asymmetry of the shape of the backward emitted emission profile is explained by an inhomogeneous spatial distribution of excited ions inside plasma volume. Near irradiated boundary of the sphere, as indicated above, concentration of excited ions is greater than ones near the shadow boundary. When the sphere is expanding (line 1), backward emitted radiation is formed by excited ions located inside the region from the irradiated boundary and to the center of the sphere. When the sphere is compressing, backward emitted radiation is formed by excited ions located between the center of the sphere and its shadow boundary, where the concentration of excited ions is lower. We also note that when substance is compressing, dashed line 3', an apparent shift of the line to the blue wing of the spectrum is observed. Such frequency shift occurs along the ray path on which the average velocity of compression of the sphere is zero.

In conclusion of this section, we again note an important conclusion: the shift of the maximum of spectral line profile to the red spectral wing region during expansion and to the blue one during compression are not caused by macroscopic motion of substance away from or toward an external observer, but it can manifest itself under the conditions of pulsating dynamics of matter.

\section{CONCLUSION}

The absorption and emitting of resonant radiation by the spherical plasma with a macroscopic motion of substance by using the method of numerical modeling have been considered. Numerical results have shown the importance of taking into account the effects of large optical thickness and Doppler shift of the absorption line profile which lead to the appearance of a specific deformation of the emission line profile. The shift of the emission frequency profile to the red wing of the spectrum for the expanding plasma, and the shift of the frequency profile to the blue wing of the spectrum for the compressing plasma were predicted. These shifts in the emission line profiles were obtained at an average velocity of ions along the ray trajectory equals to zero. Such frequency shifts will be manifested for both laboratory and cosmic plasmas. From this fact we can conclude that the shift of the spectral profile to the red or blue frequency wings for astronomical objects should not always be attributed to the moving away from the observer plasma or to approaching the observer plasma. Such spectroscopic features of the emission lines can correspond to a substance under condition of pulsating dynamics.

For spherical plasma, rotating around the axis of symmetry, absorption and emission line profiles were much wider than the corresponding line profiles in the static medium for ray tracks passing alone the sphere diameter. As the ray track was move away from the diameter of the sphere, we observed a shift of the central line frequency to the red or blue wing of the spectrum. This spectroscopic information can be used for diagnostic purposes to determine the direction of rotation of the plasma, the linear velocity of ions along the ray direction and, consequently, the period of the plasma rotation.

\section{References}

[1] C. Sack and H. Schamel, Phys. Rep. 156, 311 (1987).

[2] C. E. Simien et al, Phys. Rev. Lett. 92, 143001-1 (2004).

[3] E. A. Cummings et al, Phys. Rev. Lett. 95, 235001-1 (2005).

[4] N. R. Pereira and J. Davis, J. Appl. Phys. 64, (3), R1 (1988).

[5] D. Mihalas, Stellar Atmospheres (San Francisco: Freeman, 1978) Part 2, p 424. 
[6] E. P. Velikhov, JETP Lett. 82, 696 (2005).

[7] Y. Kikuchi, et al, Phys. Rev. Lett. 97, 085003 (2006).

[8] N. I. Kosarev, Math. Model. 18, 67 (2006).

[9] N.I. Kosarev and N.Ya. Shaparev, J. Phys. B 41, 235701 (2008).

[10] N.I. Kosarev and N.Ya. Shaparev, Doklady physics. 53, 424 (2008).

[11] N. I. Kosarev and N. Ya. Shaparev, Sov. J. Quantum Electron. 39, 1150 (2009).

[12] N. I. Kosarev and N. Ya. Shaparev, J. Phys. B 44, 195402 (2011).

[13] N.I. Kosarev and N.Ya. Shaparev, J. Phys. B 45, 165003 (2012).

[14] N.I. Kosarev, Atmospheric and oceanic optics 30, 233 (2017).

[15] N Davis, Rep. Prog. Phys. 42, 1566 (1979).

[16] N.I. Kosarev, Astrophysical bulletin 72, (2017) (to be published).

[17] N.I. Kosarev, I.M. Shkedov, Atmospheric and oceanic optics. 4 (11), 1172 (1991).

[18] N.I. Kosarev, I.M. Shkedov, Atmospheric and oceanic optics. 6 (10), 1298 (1993).

[19] N.I. Kosarev, I.M. Shkedov, Atmospheric and oceanic optics. 8 (12), 1752 (1995).

[20] N.I. Kosarev, I.M. Shkedov, Atmospheric and oceanic optics. 12 (1), 30 (1999).

[21] 3. A. K. Rzaev, Astrophysical Bulletin 67, 282 (2012). 


\section{Captions to figures}

FIG. 1. Geometry of rotating sphere plasma (a), and the template of numerical grid of the central impact plane (b) crossing the $\mathrm{XZ}$ axis.

FIG. 2. Normalized to $I_{\mathrm{S}}^{\mathrm{o}}$ spectrum of the transmitted solar radiation along the various optical tracks of the upper semicircle in Fig. 1(b). Solid line 1 corresponds to track number 2; 2-4; 3-6 and line 4 corresponds to the optical track passing along the sphere's diameter (point 7 in Fig. 1(b). Rotation is counterclockwise with velocity at the sphere's boundary equals $V_{0}=5 v_{\text {th }}=3.46 \times 10^{5} \mathrm{~m} / \mathrm{s}$. The static optical thickness of the sphere along diameter is $\tau_{0}=25$. Dashed line 4' is obtained for the same track as line 4, only for a static medium.

FIG. 3. Normalized to $I_{\mathrm{S}}^{\mathrm{o}}$ spectrum of the radiation emitted alone different optical tracks, in the direction perpendicular to the direction of sunlight of the lower semicircle in Fig. 1 (b). Solid line 1 corresponds to track number 2; 2-4; 3-7 (along the sphere's diameter). Dashed lines 1' and 2' correspond to tracks symmetrical to ones number 1 and 2, respectively, relative to the sphere's diameter. Dashed line 3' corresponds to the optical track passing along the diameter of the sphere for a static medium. Direction and velocity of rotation, the static optical thickness of the sphere, are the same as in Fig. 2.

FIG. 4. Normalized to $I_{\mathrm{S}}^{\mathrm{o}}$ spectrum of the radiation emitted alone different optical tracks, in the direction of sunlight. Solid line 1 corresponds to track number 2; 2-4;3-7 (along the sphere's diameter). Dashed lines 1' and 2' correspond to tracks symmetrical to ones number 1 and 2, respectively, relative to the Z-axis in Fig. 1 (b). Dashed line 3' corresponds to radiation propagating along the sphere's diameter for a static medium. Direction and velocity of rotation, the static optical thickness of the sphere, are the same as in Fig. 2.

FIG. 5. Relative absorption of solar radiation $I_{\mathrm{S}} / I_{\mathrm{S}}^{\mathrm{o}}$ as a function of frequency $x=\left(v-v_{0}\right) / \Delta v_{\mathrm{D}}$, in different spatial points of the sphere along its diameter: line 1 corresponds to $(r / 2 R=-0.433)$; line $2,-0.25$; line 3,0 (the centre of sphere); line $4,-0.25$; line $5,-0.433$; line $6,-0.5 . \tau_{0}=25$. $r / 2 R=-0.5$ corresponds to the irradiated boundary of the sphere, $r / 2 R=+0.5$ corresponds to the shadow boundary. The first phase of expansion, $t=1 \mu \mathrm{s}$.

FIG. 6. Normalized to $I_{\mathrm{S}}^{\mathrm{O}}$ spectrum of the emitted radiation as a function of frequency $x=\left(v-v_{0}\right) / \Delta v_{\mathrm{D}}$ : Solid line 1 corresponds to the eighth phase of expansion $(t=29 \mu \mathrm{s})$, backwards emission; 2 - the eighth phase of expansion, lateral emission; 3 - the eighth phase of expansion, forward emission. Dashed line 1 '- 3' are obtained for the eighth compression phase $(t=31 \mu \mathrm{s})$, for the backward emission, lateral and forward one, respectively. The parameters are the same as in Fig.5. 